\title{
Are Changes in MAPK/ERK Necessary or Sufficient for Entrainment in Chick Pineal Cells?
}

\author{
Geetha Yadav, ${ }^{1}$ Martin Straume, ${ }^{2}$ James Heath III, ${ }^{1}$ and Martin Zatz ${ }^{1}$ \\ ${ }^{1}$ Laboratory of Cellular and Molecular Regulation, National Institute of Mental Health, National Institutes of Health, Department of Health and Human \\ Services, Bethesda, Maryland 20892-4068, and ${ }^{2}$ Center for Biomathematical Technology, Department of Internal Medicine, University of Virginia, \\ Charlottesville, Virginia 22903
}

\begin{abstract}
Chick pineal cells in culture display a circadian rhythm of melatonin release. Light pulses can entrain (phase shift) the rhythm. One candidate for the photoentrainment pathway uses a mitogen-activated protein kinase (MAPK), also known as extracellular signalregulated kinase (ERK). We tested the hypothesis that activation of ERK (by phosphorylation to p-ERK) is necessary and/or sufficient for entrainment by measuring the ability of several drugs, light, and other perturbations to change levels of p-ERK and to induce phase shifts in the melatonin rhythm. If changes in the levels of p-ERK are sufficient for photoentrainment, then all perturbations that reduce its level must induce light-like phase shifts, and all those that increase its level must induce dark-like phase shifts. If such changes are necessary for photoentrainment, then light pulses must reduce p-ERK levels, and the duration of the light pulse, the magnitude and duration of the change in p-ERK, and the size of the phase shift must correlate. We found five perturbations that reduced p-ERK levels. Of these, two induced light-like phase shifts (PD 98059 and caffeine), one induced dark-like phase shifts (SB203580), and two did not induce phase shifts at all (U0126 and omitting a medium change). Serum increased p-ERK levels without inducing any phase shifts. Finally, light pulses did not elicit changes in p-ERK, nor was there a diurnal rhythm in p-ERK levels, nor could rapid changes in p-ERK levels have accounted for duration effects of light pulses on phase shifts. Taken together, these results argue strongly against the hypothesis that reduction (or increases) in MAPK/ERK activation is necessary or sufficient for entrainment in chick pineal cells.
\end{abstract}

Key words: MAPK; ERK; MEK inhibitor; circadian rhythms; clocks; entrainment; signal transduction; melatonin

\section{Introduction}

Chick pineal cells display a persistent, photosensitive, circadian rhythm of melatonin synthesis and release in dispersed cell culture (Deguchi, 1979). Light pulses acutely suppress melatonin output, an effect closely related to "masking," and can induce phase shifts in the rhythm, an effect closely related to entrainment of the underlying clock. The acute and phase-shifting effects of light are mediated by different mechanistic pathways (Natesan et al., 2002). Although there is some understanding of the biochemical pathway mediating the acute effect of light (Zatz, 1996; Zatz et al., 2000), there is virtually nothing known about the transduction pathway mediating the phase-shifting effects of light.

Several of the agents and perturbations that have been found to induce phase shifts in the melatonin rhythm can be viewed as cellular stressors (Takahashi et al., 1989; Zatz, 1996). One group of transduction pathways that respond to cellular stressors is the mitogen-activated protein kinase (MAPK) superfamily (Cobb,

\footnotetext{
Received July 29, 2003; revised Sept. 3, 2003; accepted Sept. 3, 2003.

We are grateful to Dr. Pierre Voisin for the gift of HIOMT antibody. We thank Dr. Stuart Dryer and Dr. Sumana Chakravarty for their very helpful procedural advice.

Correspondence should be addressed to Martin Zatz, Building 36, Room 2A-11, Laboratory of Cellular and Molecular Regulation, National Institute of Mental Health, Bethesda, MD 20892-4068. E-mail: martin.zatz@mail.nih.gov.

G. Yadav's present address: Department of Cell Biology, Neurobiology, and Anatomy, Medical College of Wisconsin, Milwaukee, WI 53226-0509.

Copyright $\odot 2003$ Society for Neuroscience $\quad$ 0270-6474/03/2310021-11\$15.00/0
}

1999; Johnson and Lapadat, 2002), also known as the extracellular signal-regulated kinase (ERK) family. These pathways are named after the protein kinases that lie at their "center." Signal transduction is mediated by a cascade of protein phosphorylations that activates one protein kinase after another until any of several specific transcription factors, including c-Fos, CREB (cAMP response element-binding protein), and Elk, become phosphorylated and activated (Su and Karin, 1996; Valjent et al., 2001). An extensive network of isoforms and parallel pathways provides flexibility and specificity (Pearson et al., 2001; Arbabi and Maier, 2002). Thus, we considered these transduction pathways to be reasonable candidates for a role in photoentrainment.

In view of the multiple, complex, and overlapping pathways in this superfamily (Mackova et al., 2000), we undertook to test the simplest hypothesis: increased (or decreased) levels of activated MAPK/ERK ( $\mathrm{p}$-ERK) are uniquely necessary and/or sufficient for entrainment of chick pineal cells in culture. If photoentrainment is mediated by reductions in the levels of activated MAPK/ERK, as has been suggested (Sanada et al., 2000), then drugs that block activation of ERK should mimic the phase-shifting effects of light pulses, and agents that promote activation of ERK should mimic the effects of dark pulses. Furthermore, light pulses should decrease levels of p-ERK, and other perturbations that induce lightlike phase shifts might do so as well. Perturbations that induce dark-like phase shifts, on the other hand, should not decrease p-ERK levels, but might instead increase p-ERK levels. To test 
these predictions, we used a number of agents that were known, expected, or unexpectedly found to alter levels of p-ERK and measured their effects on levels of p-ERK and on phase shifts of the melatonin rhythm, respectively. We also tested the effects of agents known to induce phase shifts on p-ERK levels. While this work was underway, the presence, temporal dynamics, and photoresponsiveness of MAPK/ERKs have been reported in several systems, but results related to entrainment in these different systems have been inconsistent (Obrietan et al., 1998; Sanada et al., 2000; Yokota et al., 2001; Cermakian et al., 2002). Our extensive results, taken together, argue strongly against the hypothesis positing a clear relationship between activation of MAPK/ERK and phase shifts in chick pineal cells.

\section{Materials and Methods}

Pineal cell culture. White Leghorn chicks were received $0-2 \mathrm{~d}$ after hatch from Truslow Farms (Chestertown, MD). Pineal cells were dispersed in trypsin and plated in modified McCoy's 5A medium (Invitrogen, Carlsbad, CA; 12330-031) containing 25 mM HEPES buffer, L-glutamine, penicillin, streptomycin, $10 \%$ heat-inactivated fetal bovine serum, and $10 \%$ chicken serum as described previously (Zatz et al., 1988). Each 24-well plate had cells in groups of four wells (one to four groups per plate). Experiments used cells from up to 100 glands in 60 wells, initially containing $\sim 10^{6}$ cells per well. Cells were fed by exchange of medium at least daily. Days in culture are numbered successively from the day of plating (day 1). Cells were fed with the plating medium (modified by omission of fetal bovine serum) on days 2 and 3. On day 4, they were switched to medium without serum but containing an additional $10 \mathrm{~mm}$ $\mathrm{KCl}$. The effects of feeding schedule, media, sera, and potassium on melatonin production were described previously (Zatz et al., 1988).

Light cycles. Cells were maintained at $37^{\circ} \mathrm{C}$ under $5 \% \mathrm{CO}_{2}$ in air in tissue culture incubators containing red lights, white lights, and timers as described previously (Zatz et al., 1988). They were all exposed to a cycle of $12 \mathrm{hr}$ of white light (L) and $12 \mathrm{hr}$ of red light (R), designated LR 12:12, through day 5 . In this schedule, $\mathrm{L}$ acts as "day" and, by convention, starts at zeitgeber time (ZT) 0; R acts as "night" and starts at ZT 12. In most experiments, cells were switched from LR 12:12 to constant red light (RR) before expected onset of $\mathrm{L}$ at the start of day 6 . Thereafter, media were exchanged, drugs were administered, and cells were fixed or harvested in RR unless noted otherwise.

Drugs and reagents. PD 98059 (catalog \#513000), U0126 (catalog \#662005), and SB 203580 hydrochloride (catalog \#559395) were purchased from Calbiochem (San Diego, CA). PD 98059 and U0126 were dissolved in DMSO and diluted into medium such that final DMSO concentrations were $0.1 \%$. This concentration of DMSO has no effect on either p-ERK levels or the melatonin rhythm (data not shown). SB 203580 was dissolved in sterile distilled water and diluted into medium at 1:100 or less. Caffeine (Sigma-Aldrich, St. Louis, MO; catalog \#C-0750) and $\mathrm{NaCl}$ were each dissolved directly into medium.

Mouse monoclonal antibody that specifically recognizes the activated, diphosphorylated forms of ERK 1 and ERK 2 (p-ERK) was purchased from Sigma (catalog \#M8159). Second antibody (HRP-linked antimouse IgG) was purchased from Amersham Biosciences (Piscataway, NJ; catalog \#NA 931). The rabbit polyclonal antibody against total ERK ( $t-$ ERK) (Santa Cruz Biotechnology, Santa Cruz, CA; catalog \#sc-94) recognizes both phosphorylated and nonphosphorylated forms of ERK 1 and ERK 2. Second antibody (HRP-linked anti-rabbit IgG) was purchased from Cell Signaling Technology (Beverly, MA; catalog \#7071-1).

p-ERK, t-ERK, and phospho-p38 (p-p38) standards were purchased from Cell Signaling Technology (catalog \#9103 and 9213). The p-ERK and t-ERK standards used are derived from bacterially expressed pure ERK 2 protein. Their apparent molecular weight of $42 \mathrm{kDa}$ does not change with phosphorylation. The p-p38 standard used is made from anisomycin-treated C6 glioma cell extracts. In addition to p-p38, it contains ERK $1(44 \mathrm{kDa})$ and ERK $2(42 \mathrm{kDa})$. The p-p38 cannot be distinguished in these experiments from ERK 2, because both are present in the standard and both run at an apparent molecular weight of $42 \mathrm{kDa}$. The p-p38 extract does provide, however, a distinguishable ERK 1 standard. It gives a band at $44 \mathrm{kDa}$ with the anti-p-ERK and anti-ERK antibodies that is not seen with the ERK 2 standard. SeeBlue molecular weight markers were purchased from Invitrogen (catalog \#LC 5625).

Immunoblots. Harvests for assay of p-ERK (and t-ERK) were usually performed on day 6. Medium was aspirated from the cells, and $300 \mu \mathrm{l}$ of Laemmli buffer (Sigma; catalog \#S 3401) was immediately added to each well. After $20 \mathrm{~min}$, samples were transferred to Eppendorf tubes, heated at $98^{\circ} \mathrm{C}$, and centrifuged. Aliquots from each well containing $\sim 10 \mu \mathrm{g}$ of protein were loaded onto a 10\% Bis-Tris gel (Invitrogen; catalog \#NP 0302) and subjected to PAGE. Proteins were transferred from the gel to a nitrocellulose membrane. Membranes were blocked with Tris-buffered saline containing $0.1 \%$ Tween 20 and 5\% fat-free dry milk. For determination of p-ERK, membranes were incubated with anti-p-ERK antibody (1:2000) at $4^{\circ} \mathrm{C}$ overnight, washed three times at room temperature, and incubated for $1 \mathrm{hr}$ with secondary antibody (1:2000). Membranes were washed, and then bound secondary antibody was visualized using a commercial chemiluminescence detection kit (Pierce, Rockford, IL; catalog \#34080) according to the manufacturer's instructions. Bands were quantified using a Kodak Image Station (PerkinElmer Life Sciences, Boston, MA; model 440CF). For determination of t-ERK, the anti-ERK antibody was used at 1:5000 and its corresponding secondary antibody was used at 1:2000. In early experiments, t-ERK determinations used separate gels. In most experiments, however, membranes were stripped after determination of p-ERK (using 0.2 M glycine buffer, $\mathrm{pH} 2.8,15 \mathrm{~min}$ ), blocked, and then reprobed for determination of t-ERK.

Calculation of normalized $p$-ERK/t-ERK ratios. Early experiments gave a troublesome degree of variability in density readings. To compensate, an aliquot of sample from each control well was run on all gels, and values for experimental bands were normalized to the average value of the four controls. Values of t-ERK were similarly normalized to controls. A normalized p-ERK/t-ERK ratio was calculated from these values. Differences between experimental and control groups were analyzed using Student's $t$ test.

Assay of ${ }^{14} \mathrm{C}$-melatonin release. $\mathrm{L}-\left[\right.$ side chain- $\left.3-{ }^{14} \mathrm{C}\right]$-tryptophan (specific activity $53.8-54.6 \mathrm{mCi} / \mathrm{mmol}$ ) was purchased from PerkinElmer Life Sciences (catalog \#NEC-367). McCoy's 5A medium contains $\sim 0.03$ mM tryptophan (of which more than half is contributed by the Bactopeptone, a proprietary nutrient digest, that it contains). Cells were exposed to ${ }^{14} \mathrm{C}$-tryptophan for $24 \mathrm{hr}$ before the start of timed collections. Different experiments used $0.45-0.50 \mu \mathrm{Ci} / \mathrm{ml}$, present from day 5 . Media were collected and replaced at $4 \mathrm{hr}$ intervals in RR (unless noted otherwise) starting on day 6 . Spent medium from each well was transferred into a polypropylene test tube containing $0.05 \mathrm{ml}$ of an indole carrier mix, extracted into $5 \mathrm{ml}$ of chloroform, and backwashed with acid and base as described previously (Zatz et al., 1988). Three milliliters of the final chloroform phase were transferred to scintillation vials, dried, and counted.

Calculation of phase shifts. ${ }^{14} \mathrm{C}$-melatonin rhythm data were analyzed by nonlinear least squares regression (Johnson and Frasier, 1985; Straume et al., 1991) to a cosine function, from which were estimated parameter values for oscillatory amplitude, period, and acrophase, as well as terms for constant offset and linear trend with time (slope). Approximate nonlinear asymmetric joint parameter confidence limits were subsequently evaluated at $68.26 \%$ confidence probability (the probability of $1 \mathrm{SD}$ ). Of the 15 points available in each data set, only the last 9 were analyzed (from 28 to $60 \mathrm{hr}$ after release into RR). Acrophase was estimated for both the first and second occurrences of acrophase in the analyzed time series (second and third peaks in the melatonin data). Phase and period were computed as variance-weighted averages with associated variance-weighted SEs of the mean. Phase shifts were computed as differences between control and experimental values. SEs for the shifts took the SEs for the respective phases into account, and statistical significance was determined using Student's $t$ test.

Immunocytochemistry. Cells were fixed in their wells in $4 \%$ paraformaldehyde on day 3 of culture, washed, blocked in PBS containing $0.5 \%$ Triton X-100 and 3\% sheep serum, and incubated overnight with primary antibody at $4^{\circ} \mathrm{C}$. For double-labeling experiments, a mouse monoclonal antibody directed against t-ERK (Santa Cruz Biotechnology; cat- 

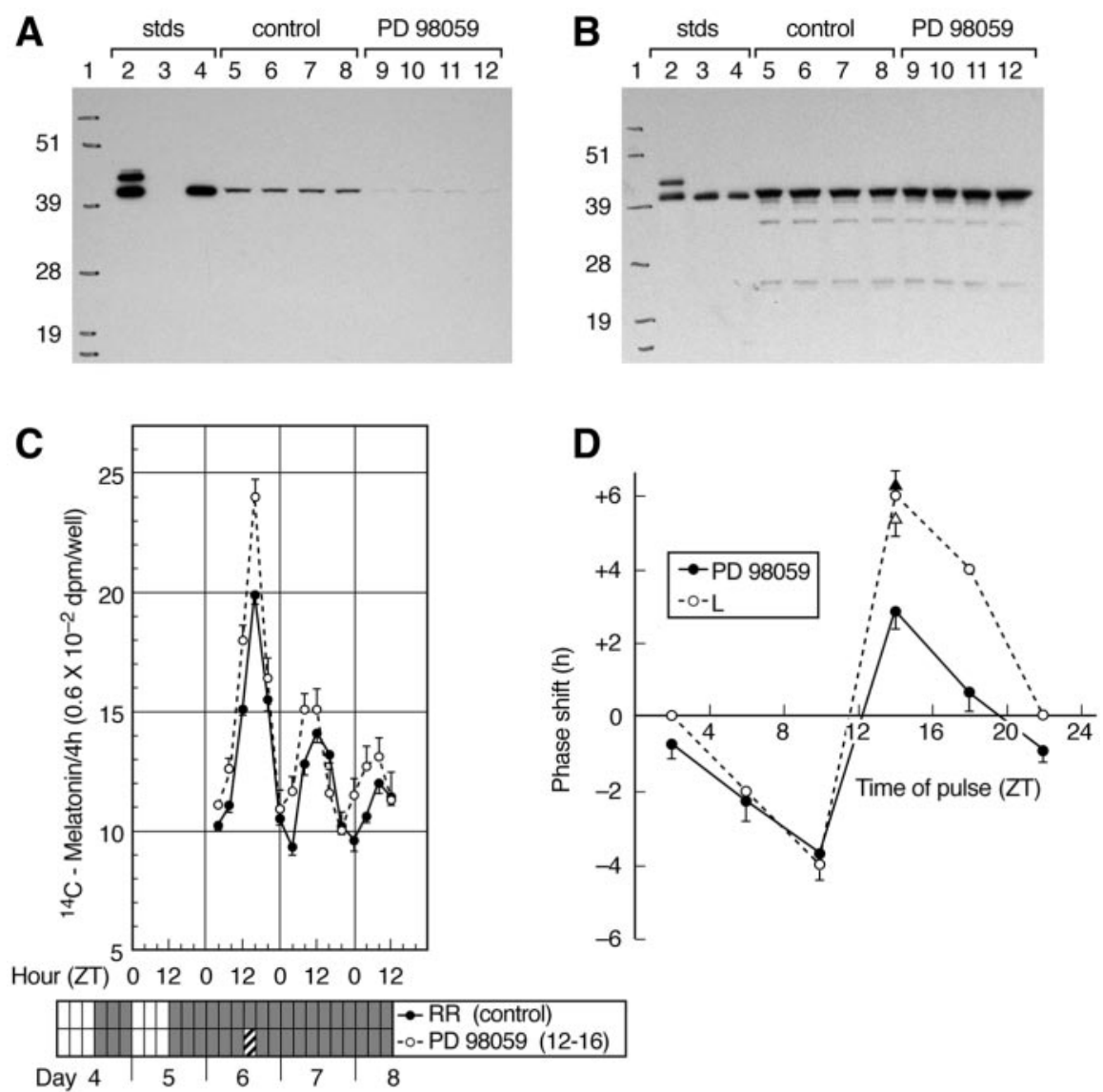

Figure 1. Effects of PD 98059 on ERK and on the melatonin rhythm. $A$, Exposure to PD 98059 from ZT 12 to 16 reduced levels of p-ERK. Chick pineal cells were plated on day 1 and maintained in a cycle of $12 \mathrm{hr}$ white light and $12 \mathrm{hr}$ red light through day 5 . They were switched to constant red light before the start of day 6. 0n day 6, cells in one group of four wells were exposed (in R) to $10 \mu \mathrm{m}$ PD 98059 for 4 hr, from ZT 12 to 16. Control cells also had their medium changed at ZT 12. Experimental and control cells were harvested at ZT 16 in R, extracted, and subjected to PAGE and immunoblot analysis as described in Materials and Methods. Lane 1 contained SeeBlue molecular weight markers; lanes 2- 4 had p-p38, t-ERK, and p-ERK standards, respectively. Lanes 5-8 contained an aliquot of the sample from each control well, respectively, and lanes $9-12$ contained an aliquot of the sample from each well exposed to PD 98059. B, PD 98059 did not change levels of t-ERK; same samples and procedure as for p-ERK $(A)$. Lane 1 contained molecular weight markers; lanes 2-4 had p-p38, t-ERK, and p-ERK standards, respectively. Lanes 5- 8 contained an aliquot of the sample from each control well, respectively, and lanes 9-12 contained an aliquot of the sample from each well exposed to PD 98059. In contrast to other experiments, this experiment assayed samples of t-ERK on a separate gel rather than after stripping the membrane used for p-ERK. C, Exposure to PD 98059 from ZT 12 to 16 evoked a phase advance in the melatonin rhythm. Chick pineal cells in primary culture were maintained under LR 12:12 through day 5 and then switched to RR. On day 6, cells in one group of four wells were exposed (in R) to $10 \mu \mathrm{M}$ PD 98059 for $4 \mathrm{hr}$, from ZT 12 to 16. Control cells had their media collected and replaced as usual. All cells were in medium containing ${ }^{14} \mathrm{C}$-tryptophan from day 5 onward. Media were collected (and replaced) every $4 \mathrm{hr}$ in RR from the start of day 6 and then assayed for ${ }^{14} \mathrm{C}$-melatonin as described in Materials and Methods. Solid lines and closed circles represent results from controls, and dotted lines and open circles represent results from cells treated with PD 98059. Each point is the mean \pm SE of data from four wells, shown at time of collection. Bar at the bottom shows the lighting conditions: open intervals were in L, stippled intervals were in $\mathrm{R}$, and the hatched interval indicates when the drug was present. ${ }^{14} \mathrm{C}$-tryptophan was used at $0.49 \mu \mathrm{Ci} / \mathrm{ml}$, and blanks (not subtracted) gave $73 \pm 2 \mathrm{dpm}$ per well $(n=12)$. $D$, The phase response curve for PD 98059 is similar to that for light pulses. Phase shifts evoked by $4 \mathrm{hr}$ pulses of $10 \mu \mathrm{M}$ PD 98059 are plotted against the time at which the drug was administered. Phase-shift values are shown at the midpoint of the $4 \mathrm{hr}$ treatment intervals. Phase advances are depicted, by convention, as positive shifts, and phase delays are depicted as negative shifts. Solid lines and closed circles represent phase shifts induced by $4 \mathrm{hr}$ pulses of $10 \mu \mathrm{M}$ PD 98059, and dotted lines and open circles represent phase shifts induced by $4 \mathrm{hr}$ pulses of light for comparison [taken from Zatz et al. (1988)]. Each point on the curves represents the phase shift determined from a separate experimental group; the PD 98059 point shown at ZT 14 was determined from the results of the experiment shown in C. Other points represent phase shifts determined in similar experiments, but in which the drug was administered at other times. The dark triangle represents the phase shift induced by $30 \mu \mathrm{m}$ PD 98059 at ZT 12-16, and the open triangle denotes the phase shift induced by $L$ in the same experiment. Each point is the mean \pm SE of data from four wells.

alog \#sc-1647) was used at 1:200, and a rabbit polyclonal antibody directed against hydroxyindole-O-methyltransferase (HIOMT), provided by Dr. Pierre Voisin (Laboratoire de Neurobiologie Cellulaire, Poitier, France), was used at 1:2000 (Voisin et al., 1988). Wells were washed in PBS containing 0.5\% Triton X-100 and then incubated with Alexa Fluor 488 against mouse (Molecular Probes, Eugene, OR; catalog \#A11001) and Alexa Fluor 594 against rabbit (Molecular Probes; catalog \#A11012), each at 1:500. Wells were again washed with PBS containing $0.5 \%$ Triton X-100 and then examined under the microscope.

\section{Results}

\section{PD 98059 reduced levels of p-ERK and} induced light-like phase shifts

PD 98059 is a potent and specific inhibitor of MAPK kinase 1 (MEK) and prevents it from phosphorylating and activating MAPK/ERK (Alessi et al., 1995; Dudley et al., 1995; Davies et al., 2000). Treatment with $10 \mu \mathrm{M}$ PD 98059 for $4 \mathrm{hr}$, between ZT 12 and 16 in RR, reduced levels of p-ERK by $95 \%$ in the experiment shown in Figure $1 A$ (normalized $\mathrm{p}$-ERK/t-ERK $=0.05 \pm$ 0.01 relative to controls; $p<0.001)$. Similar but somewhat weaker effects were obtained in five other experiments with the same design. Pooled results from the six experiments gave a ratio of $\mathrm{p}$-ERK to t-ERK of $24 \pm 7 \%$ relative to that in controls $(p<0.001)$. PD 98059 did not affect levels of total ERK (t-ERK) at all ( $97 \pm 3 \%$ of controls) (Fig. $1 B$ ). Levels of t-ERK from experimental wells remained essentially the same as those from control wells in this and all other experiments.

In samples from chick pineal cells, a single band was detected with either antip-ERK (Fig. $1 A$ ) or anti-t-ERK antibodies (Fig. $1 B$ ), which migrated with the $42 \mathrm{kDa}$ ERK 2 standard. The ERK 1 isoform was visible in the cell extracts used as standards for p-p38 but was not detected in the chick pineal cells.

The effect of $10 \mu \mathrm{M}$ PD 98059 on p-ERK levels was essentially the same when tested at the opposite phase (ZT $0-4)$. The drug reduced normalized p-ERK levels to $17 \pm 1 \%$ of controls $(p<$ $0.001)$. At a somewhat higher concentration, $30 \mu \mathrm{M}$, there was a similar reduction; normalized p-ERK levels were $19 \pm 4 \%$ of controls $(p<0.001)$. The effect of PD 98059 was rapid and sustained: p-ERK levels had fallen to $30 \pm 2 \%$ of controls ( $p<$ 0.001 ) after $10 \mathrm{~min}$ of treatment with 10 $\mu \mathrm{M}$ (data not shown) and stayed down for at least $4 \mathrm{hr}$ (see above).

We tested the effects of $4 \mathrm{hr}$ pulses of PD 98059 on the melatonin rhythm. Treatment of the cells with $10 \mu \mathrm{M}$ PD 98059 from ZT 12-16 induced a phase advance of the melatonin rhythm (Fig. 1C) of $+2.86 \pm 0.48 \mathrm{hr}(p<0.05)$. Similar results were obtained in two other experiments. Pooled results from the three experiments gave a phase advance of $+2.78 \pm 0.34 \mathrm{hr}(p<0.05)$. Light pulses 
at this phase also induce phase advances (Zatz et al., 1988; Takahashi et al., 1989). Indeed, the relationship between the phase shifts induced and the time of the pulse [plotted in the phase response curve (PRC)] was very similar for $4 \mathrm{hr}$ pulses of light and $4 \mathrm{hr}$ pulses of PD 98059 (Fig. 1 D). Both perturbations had little or no effect on subsequent phase when administered in the morning (ZT $0-4)$, induced increasingly large delays when administered later in the day (ZT 8-12), and then switched suddenly to inducing phase advances when given during early night (ZT 12-16). The phase shifts induced by $10 \mu \mathrm{M}$ PD 98059 were smaller than those induced by light. A higher concentration of PD 98059 (30 $\mu \mathrm{M})$, however, evoked a phase advance at ZT 12-16 approximately twice the size of that evoked by $10 \mu \mathrm{M}$ (Fig. $1 D$ ). This phase shift was comparable in magnitude to that evoked by light. The marked increase in the size of the phase shift evoked by $30 \mu \mathrm{M}$ PD 98059 as compared with $10 \mu \mathrm{M}$ did not correspond to a marked increase in the effect of the drug on levels of p-ERK (see above). Light pulses also induce an acute decrease in melatonin secretion. PD 98059 did not have any appreciable acute effect on melatonin output (Fig. 1C).

\section{U0126 reduced levels of p-ERK but did not induce phase shifts}

U0126 is another inhibitor of MEK, mechanistically similar to PD 98059 but more potent in blocking activation of ERK (Favata et al., 1998). In our hands, exposure of cells to $10 \mu \mathrm{M}$ U0126 between ZT 12 and 16 (Fig. $2 A$ ) reduced p-ERK to virtually undetectable levels (normalized p-ERK/t-ERK ratio $0.02 \pm 0.004 ; p<$ 0.001 ). The same result was obtained in three other experiments using the same design. Pooled results from the four experiments gave a ratio of $\mathrm{p}$-ERK to t-ERK of $1 \pm 1 \%$ relative to that in controls $(p<0.001)$. Complete blockade was also observed when the drug was tested at the opposite phase (ZT $0-4)$. The effect of $10 \mu \mathrm{M}$ U0126 was also rapid and sustained; p-ERK levels fell to $5 \pm 1 \%$ of controls $(p<0.001)$ after $10 \mathrm{~min}$ treatment and remained undetectable for at least $4 \mathrm{hr}$ (see above). U0126, like PD 98059, did not change levels of t-ERK.

We tested the effects of $4 \mathrm{hr}$ pulses of $10 \mu \mathrm{M} \mathrm{U} 0126$ on the melatonin rhythm. In contrast to PD 98059, U0126 from ZT 12-16 induced no phase shift in the melatonin rhythm (Fig. 2B) (phase shift $=+0.76 \pm 0.75 \mathrm{hr}$ ). Similar results were obtained in three other experiments (pooled results from the four experiments: $+0.81 \pm 0.33 \mathrm{hr}$ ). Nor did treatment with U0126 induce phase shifts at other phases. Pooled results from three experiments with $10 \mu \mathrm{M} \mathrm{U} 0126$ at ZT $0-4$ gave a phase shift of $-0.19 \pm$ $0.45 \mathrm{hr}$, and from two experiments at ZT $8-12$ gave $+0.10 \pm 0.53$ hr. Treatment with U0126, like treatment with PD 98059, had no acute effect on melatonin production.

\section{SB203580 reduced levels of p-ERK and induced dark-like phase shifts}

SB203580 is a putatively specific inhibitor of the activity of p38 kinases (not of their phosphorylation), which are parallel members of the MAPK superfamily and are activated by cellular stressors (Cuenda et al., 1995). We tested three different concentrations of SB203580 for effects on p-ERK levels (Fig. 3A). At $10 \mu \mathrm{M}$ SB 203580, 4 hr pulses at ZT $0-4$ had a small effect (normalized $\mathrm{p}$-ERK/t-ERK ratio $=0.87 \pm 0.03 ; p<0.05)$. Strong reductions were seen, however, at higher concentrations. At $30 \mu \mathrm{M}, \mathrm{SB}$ 203580 reduced the ratio to $0.53 \pm 0.06(p<0.001)$ and at 100 $\mu \mathrm{M}, \mathrm{SB} 203580$ reduced the ratio to $0.09 \pm 0.01(p<0.001)$. A similar effect and concentration dependence were observed when
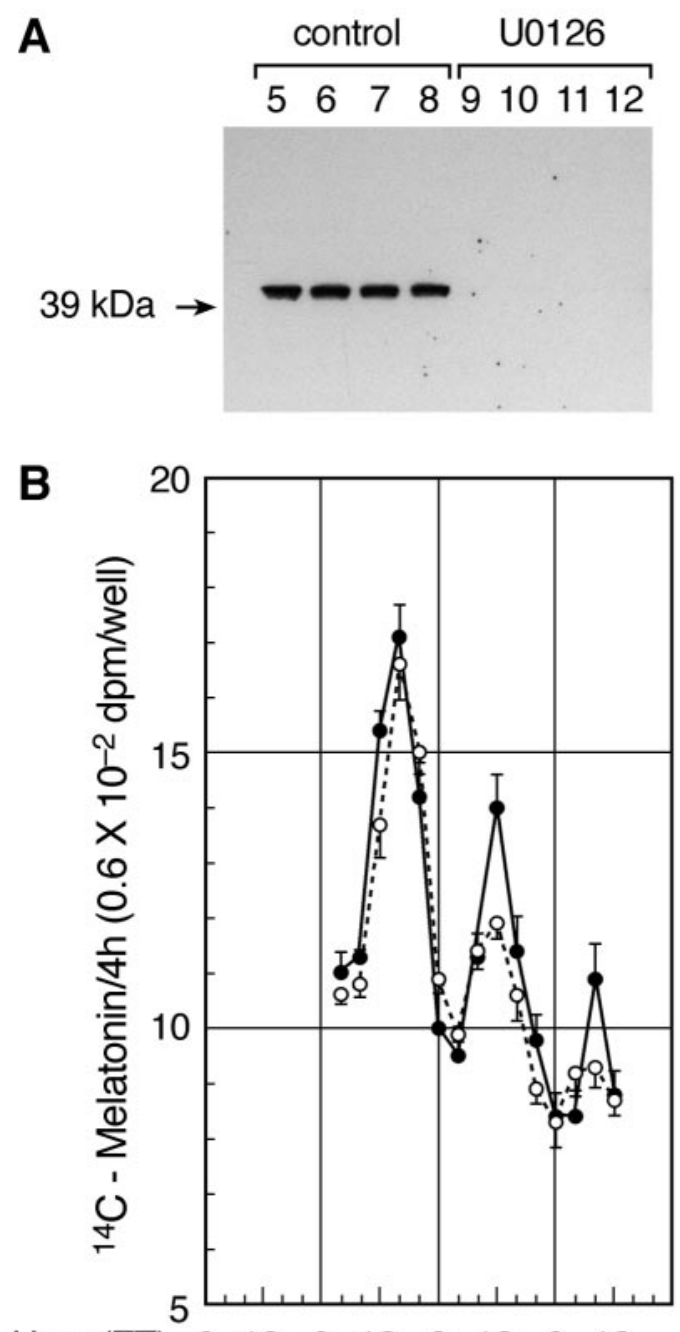

Hour (ZT) $0 \begin{array}{llllllll}12 & 0 & 12 & 0 & 12 & 0 & 12\end{array}$

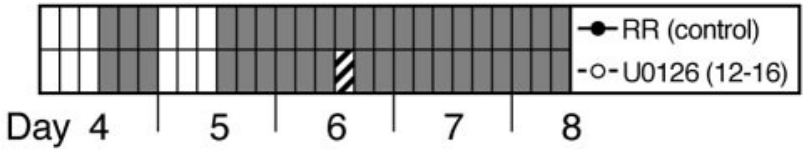

Figure 2. Effects of U0126 on ERK and on the melatonin rhythm. A, Exposure to U0126 from ZT 12 to 16 reduced levels of p-ERK; experimental design and procedures as in Figure $1 A$. Lanes 1-4 (data not shown) contained molecular weight markers and standards. Lanes 5-8 contained samples from the control group, and lanes 9-12 contained samples from the group exposed to U0126 (10 $\mu \mathrm{M})$ from ZT 12 to 16 . Each sample is from a separate well ( $n=4$ wells per group) collected at ZT 16. B, Exposure to U0126 from ZT 12 to 16 did not evoke a phase advance in the melatonin rhythm; experimental design and procedures as in Figure 1C. One group of cells was exposed to U0126 (10 $\mu \mathrm{m}$ ) from ZT 12 to 16. Solid lines and closed circles represent results from controls, and dotted lines and open circles represent results from cells treated with U0126. Each point is the mean \pm SE of data from four wells, shown at time of collection. Medium contained $0.48 \mu \mathrm{Ci} / \mathrm{ml}^{14} \mathrm{C}$-tryptophan, and blanks (not subtracted) were $103 \pm 3 \mathrm{dpm}$ per well $(n=12)$.

the drug was tested at the opposite phase (ZT 12-16; data not shown).

We compared the concentration dependence of the effects of SB 203580 on p-ERK levels with that of its effects on the melatonin rhythm (Fig. 3A). At $1 \mu \mathrm{M}, \mathrm{SB} 203580$ evoked no visible phase shift $(-0.31 \pm 0.89 \mathrm{hr})$. At $10 \mu \mathrm{M}$, it induced a significant phase delay $(-3.86 \pm 0.83 \mathrm{hr} ; p<0.05)$, despite a relatively modest reduction in p-ERK levels. It should be noted, however, that phase delays evoked at ZT $0-4$ are characteristic of dark pulses, 
A
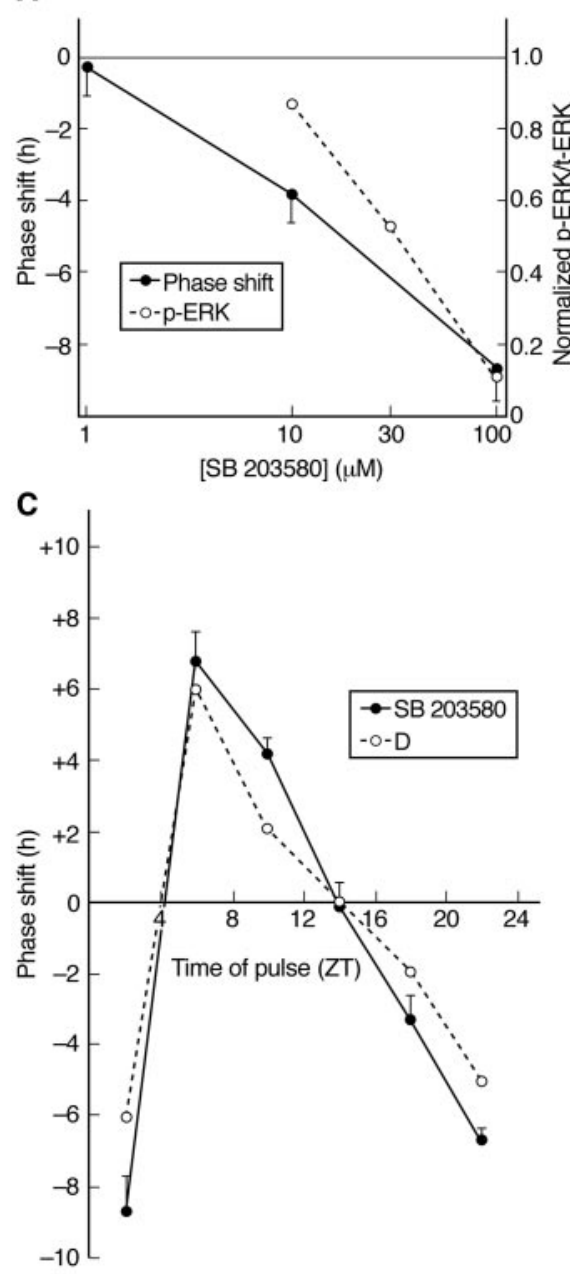

Figure 3. Effects of SB 203580 on ERK and on the melatonin rhythm. A, Comparison of concentration dependence of effects of SB 203580 on p-ERK and on the melatonin rhythm. Several concentrations of SB 203580 were tested for effects on p-ERK levels and, in separate experiments, on the melatonin rhythm. Designs were similar to those used for Figure 1, $A$ and $C$, respectively, except that drug exposure occurred from ZT 0 to 4 . Solid lines and closed circles represent phase shifts, and dotted lines and open circles represent relative levels of $p$-ERK. Means $\pm S E$ are shown; SEs for $p$-ERK fell within the points drawn. Decreasing (more negative) values for phase shifts indicate phase delays of increasing magnitude. Decreasing values for $p$-ERK indicate greater reduction in relative $p$-ERK levels. $B$, Exposure to SB 203580 from ZT 0 to 4 evoked a phase delay in the melatonin rhythm. Experimental design is as in Figure 1C, except for the time of drug treatment. One group of cells was exposed to $100 \mu \mathrm{m}$ SB 203580 from ZT 0 to 4 . Solid lines and closed circles represent results from controls, and dotted lines and open circles represent results from cells treated with SB 203580. Each point is the mean \pm SE of data from four wells, shown at time of collection. Medium contained $0.50 \mu \mathrm{Ci} / \mathrm{ml}^{14} \mathrm{C}$-tryptophan, and blanks (not subtracted) were $124 \pm 4 \mathrm{dpm}$ per well $(n=12)$. C, The phase response curve for SB 203580 is similar to that for dark pulses. Phase shifts evoked by $4 \mathrm{hr}$ pulses of $100 \mu \mathrm{M}$ SB 203580 (solid lines and closed circles) are plotted against the time at which the drug was administered. Derivation is as in Figure 1D. Dotted lines and open circles represent phase shifts induced by dark pulses for comparison [taken from Zatz et al. (1988)]. Phase advances are depicted as positive shifts, and phase delays are depicted as negative shifts. Each point represents the phase shift determined from a separate experimental group in an experiment similar to that shown in $B$ but with the drug added at different times. Each point is the mean $\pm S E$ of data from four wells.

not light pulses. At $100 \mu \mathrm{M}, \mathrm{SB} 203580$ induced a large dark-like phase delay of $-8.69 \pm 0.97 \mathrm{hr}(p<0.001)$, even though normalized p-ERK/t-ERK ratios were markedly reduced (Fig. $3 A$ ). Data from another experiment with the same design are shown in Figure $3 B$, with a phase delay of $-6.89 \pm 0.09 \mathrm{hr}(p<0.001)$. Like the other drugs tested, SB 203580 had little acute effect on melatonin secretion (Fig. 3B).

We compared the PRC for $4 \mathrm{hr}$ dark pulses with the PRC for 4 hr pulses of $100 \mu \mathrm{M}$ SB 203580 (Fig. 3C). They are indistinguishable. Maximal phase delays are induced in the morning (ZT $0-4$ ) and then switch suddenly to maximal phase advances in the next interval (ZT 4-8) that then decrease to little or no effect in the evening (ZT 12-16) and finally become increasing delays to the next morning.

It can be seen from a comparison of Figures $1 D$ (the light-like PRC) and $3 C$ (the dark-like PRC) that by testing perturbations at the two phases ZT $0-4$ and ZT $12-16$ we could infer whether agents were likely to have light-like, dark-like, or no effects on subsequent phase. PD 98059 reduced p-ERK levels and had light-like phase shifting effects. At $100 \mu \mathrm{M}$, SB 203580 reduced p-ERK levels at least as much as did PD 98059, yet this reduction did not interfere with, or reverse, its darklike phase-shifting effects.

\section{Chicken serum increased levels of p-ERK but did not induce phase shifts} All of the agents tested above reduced the levels of activated ERK. Serum is one agent known to increase the levels of p-ERK in some systems (Mitra et al., 1993; Troppmair et al., 1994). Addition of equal volumes of chicken serum to the standard medium (i.e., $50 \%$ chicken serum) also increased levels of $\mathrm{p}$-ERK in the chick pineal cells (Fig. 4A). Normalized p-ERK/t-ERK ratios were $188 \pm 6 \%$ of controls $(p<$ 0.001 ) after $4 \mathrm{hr}$ treatment from ZT 0 to 4. Similar results were obtained using $20 \%$ chicken serum at ZT $0-4$ or $50 \%$ chicken serum at the opposite phase (ZT 12-16) or $50 \%$ horse serum at ZT $12-16(150-174 \%$ of controls; all $p<0.001$ ). The effect of $50 \%$ chicken serum was fast and sustained; the increase after $15 \min (179 \pm 5 \%$ of controls; $p<0.001$ ) was similar to that after $4 \mathrm{hr}$. t-ERK levels remained unchanged.

We tested the effects of $4 \mathrm{hr}$ pulses of serum on the melatonin rhythm. Treatment of the cells with $50 \%$ chicken serum from ZT 0 to 4 induced no dark-like phase delay in the experiment shown in Figure $4 B$ (phase shift $=-0.65 \pm 0.67 \mathrm{hr}$ ) or in another with the same design (phase shift $=+0.10 \pm 0.64 \mathrm{hr}$ ). Nor was there a light-like phase advance when cells were exposed to $50 \%$ chicken serum from ZT 12 to 16 (two experiments; phase shift $=$ $+0.28 \pm 0.48 \mathrm{hr}$ ). Treatment of the cells with $50 \%$ horse serum and $20 \%$ chicken serum, respectively, also had no effect on subsequent phase.

\section{High osmolarity and caffeine induced light-like phase shifts but had different effects on p-ERK}

We tested two perturbations that are known to induce light-like phase shifts in the melatonin rhythm, high concentrations of caffeine (Zatz and Heath, 1995) and high osmolarity (Zatz and Wang, 1991), for their effects on p-ERK levels. Treatment of cells with caffeine (5 mM) at ZT 12-16 did reduce levels of p-ERK (normalized $\mathrm{p}$-ERK/t-ERK ratios from two experiments were 
A

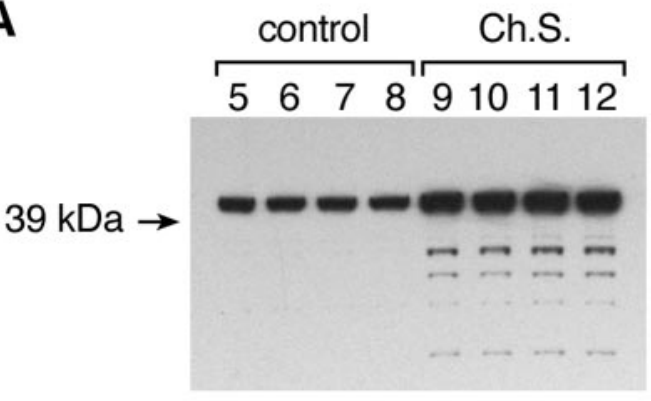

B

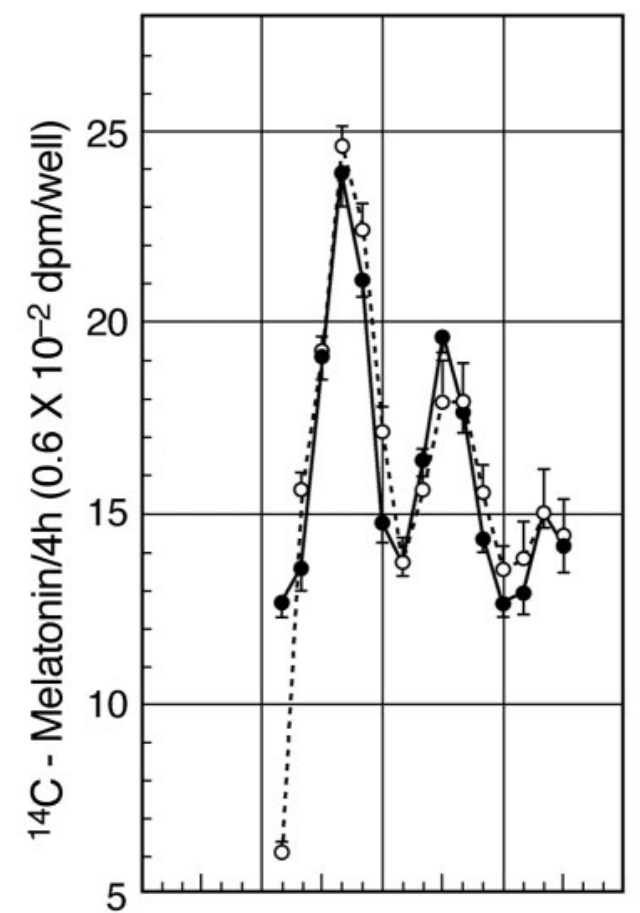

Hour (ZT) $0 \begin{array}{lllllll}12 & 0 & 12 & 0 & 12 & 0 & 12\end{array}$

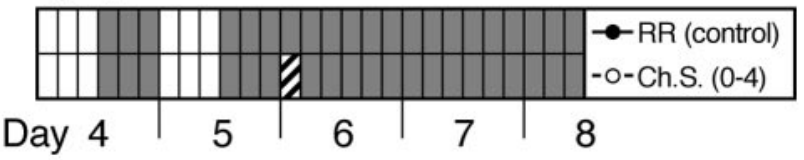

Figure 4. Effects of chicken serum on ERK and on the melatonin rhythm. $A$, Exposure to $50 \%$ chicken serum from ZT 0 to 4 increased levels of p-ERK. Experimental design is as in Figure $3 A$, with one group exposed to 50\% chicken serum from ZT 0 to 4 . Lanes 1-4 (data not shown) contained molecular weight markers and standards. Lanes 5-8 contained samples from the control group, and lanes 9-12 contained samples from the group exposed to serum. Each sample is from a separate well ( $n=4$ wells per group) collected at ZT 4. B, Exposure to $50 \%$ chicken serum from ZT 0 to 4 did not induce phase shifts in the melatonin rhythm. Experimental design is as in Figure $3 B$, with one group exposed to $50 \%$ chicken serum from ZT 0 to 4 . Solid lines and closed circles represent results from controls, and dotted lines and open circles represent results from cells treated with serum. Each point is the mean $\pm S E$ of data from four wells, shown at time of collection. Medium contained $0.49 \mu \mathrm{Ci} / \mathrm{ml}{ }^{14} \mathrm{C}$-tryptophan, except during exposure to the serum. Consequently, there was a marked reduction in apparent ${ }^{14} \mathrm{C}$-melatonin output during the serum pulse on day 6 (the first point shown). Apparent melatonin output recovered in the next interval when the "normal" ${ }^{14} C$-tryptophan was restored. Blanks (not subtracted) were $85 \pm 4 \mathrm{dpm}$ per well $(n=12)$.

$0.56 \pm 0.06 ; p<0.01)$ although not as much as did $10 \mu \mathrm{M}$ PD 98059 or U0126. Addition of $50 \mathrm{~mm} \mathrm{NaCl}$ at ZT 12-16, however, did not affect levels of p-ERK (normalized p-ERK/t-ERK ratios from two experiments were $0.96 \pm 0.05)$. Thus, changes in p-ERK cannot be considered necessary for, or characteristic of, light-like phase shifts. Absent a hypothesis for the site of conver- gence of the entrainment pathways for these agents with that for light, their interactions with ERK were not pursued further, nor were those of other perturbations known to induce phase shifts in the melatonin rhythm.

\section{Medium exchange transiently increased levels of p-ERK but its omission did not induce phase shifts}

Most of the p-ERK experiments reported above used $4 \mathrm{hr}$ time points. It mattered little whether p-ERK values were compared with zero time controls or controls whose medium had been changed $4 \mathrm{hr}$ earlier, because values for these different controls were essentially the same. Pooled results from four experiments gave $1.09 \pm 0.03$ for the $4 \mathrm{hr}$ /zero time ratio. It also did not matter when short-term effects of perturbations were tested at a single time point, because control groups always had their medium changed at the same time as the experimental groups and were harvested after the same interval. When we started doing time courses, however, e.g., for light pulses, we noted grossly inconsistent values in the normalized data from experiment to experiment, which we ultimately traced to differences in the interval between medium change and harvest of the experimental groups and controls. On testing the effects of medium change alone, we noted a rapid, marked, and transient increase in the levels of p-ERK relative to "zero time" controls, whose medium was not changed (Fig. 5A). Relative p-ERK levels rose rapidly, in $\sim 5 \mathrm{~min}$, to a peak that was almost sixfold that of controls (normalized $\mathrm{p}-\mathrm{ERK} / \mathrm{t}-\mathrm{ERK}=5.91 \pm 0.04 ; p<0.001)$ and then declined back toward control values, reaching them within $3 \mathrm{hr}$ (normalized $\mathrm{p}$-ERK/t-ERK $=1.07 \pm 0.05)$. Thus, values for short times after intentional perturbations could not all be compared with a single control. We considered whether these effects of medium change were based on differences in $\mathrm{pH}$, temperature, nutrients, etc., in the fresh medium that was added relative to the "spent" medium that was removed. Attempts to isolate these variables (compared with no change in medium) all gave similar, positive results. Finally, we simply removed the "spent" medium and put it back again and still got the same increase as with fresh medium (data not shown). Evidently the mechanical agitation of ordinary medium exchange is sufficient to raise p-ERK levels.

Our protocol for monitoring the rhythm of melatonin release requires collecting and replacing the radioactive medium every 4 hr. The increase in relative p-ERK levels after medium change alone provides a novel perturbation with which to test the effects of lowering p-ERK levels on the melatonin rhythm. By not changing the medium at a certain time, the ensuing increase would not occur. Indeed, relative to the normal procedure of collecting and replacing the medium, omitting the medium change results in a rapid, marked, and transient decrease in p-ERK levels (Fig. 5A, inset). In contrast to the effects of the previous perturbations tested, the relative decrease, in the cells with no medium change, is transient: greatest in the first minutes and almost over within $1 \mathrm{hr}$.

We tested the effects of omitting the normal medium change on the melatonin rhythm (Fig. 5B). Omitting the medium change at ZT 12 induced no phase shift in the melatonin rhythm (phase shift $=+0.01 \pm 0.68 \mathrm{hr}$ ). Similar results were obtained in another experiment $(-0.23 \pm 0.67 \mathrm{hr})$. Omitting the normal medium change at ZT 0 also had no effect on subsequent phase $(-0.08 \pm 0.59 \mathrm{hr})$.

\section{Light did not change levels of p-ERK but did induce duration-dependent phase shifts}

We then asked whether exposure to white light changes levels of p-ERK in these cells (Fig. 6A). Matched controls were used to 


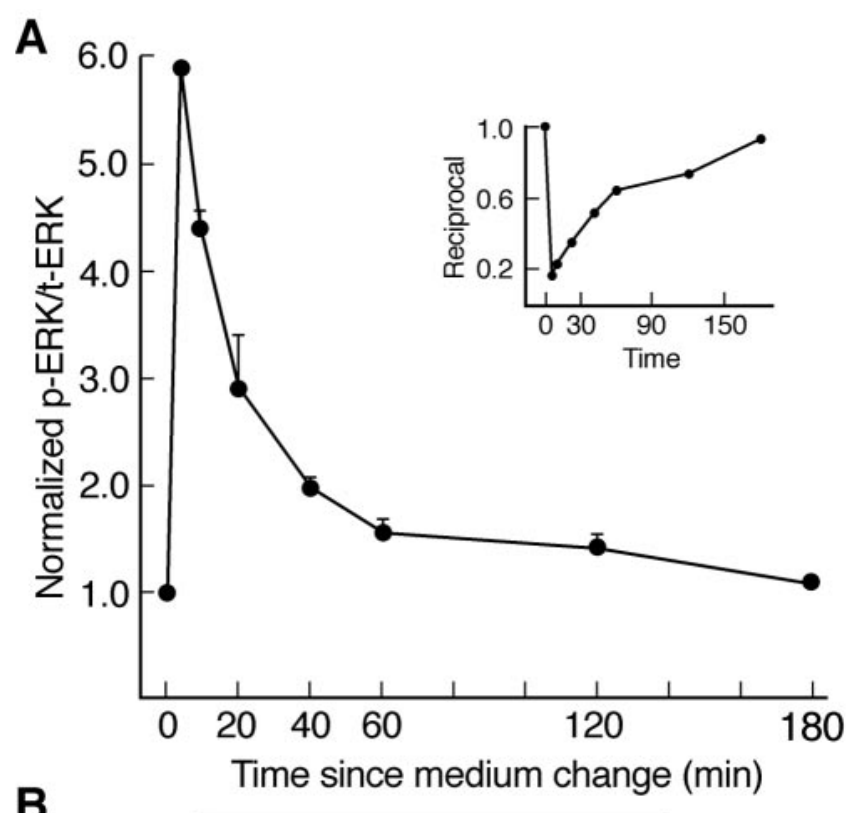

B

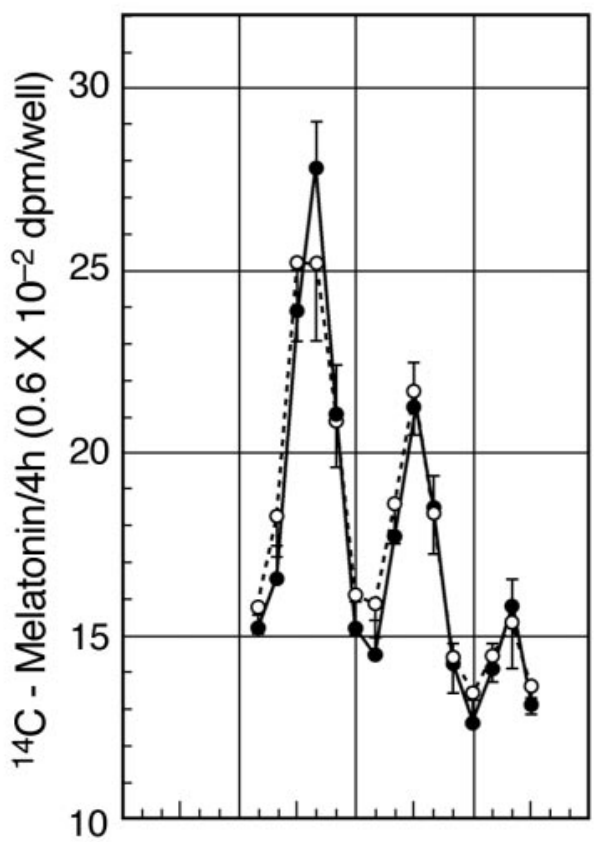

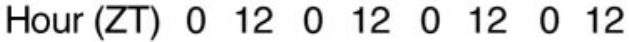

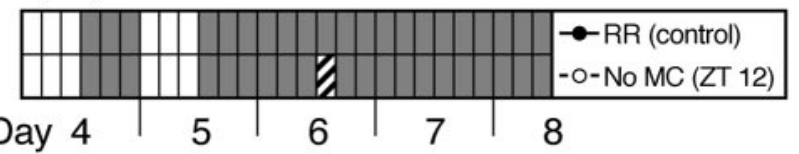

Figure 5. Effects of medium change on ERK and on the melatonin rhythm. A, Medium change strongly, but transiently, increased levels of $p$-ERK. Cells were maintained under $L R$ 12:12 through day 5 and then switched to RR. Between ZT 13 and 16, different groups had their medium changed, i.e., replaced with fresh medium, at different times. Groups of cells were then harvested $5,10,20,40,60,120$, and 180 min after their medium change, all close to ZT 16. The control group did not have its medium changed and was also harvested at ZT 16. Each point is the mean $\pm S E$ of data from four wells. Note that medium change caused a rapid, marked, and transient increase in $p$-ERK levels relative to cells whose medium was not changed. Inset, p-ERK values for the same experiment using reversed ratios, i.e., the groups with medium change here being treated as the controls. Reciprocal values of those shown in $A$ are plotted. Note that cells with medium that was not changed showed a rapid, marked, and transient decrease in p-ERK levels relative to cells whose medium was changed. This provides the basis for the experiment shown in $B$. B, Omitting the medium change at ZT 12 did not evoke a phase advance in the melatonin rhythm. Experimental design is similar to that in Figure $1 C$, except that the perturbation at ZT 12 was omission of the usual medium change. Consequently, there
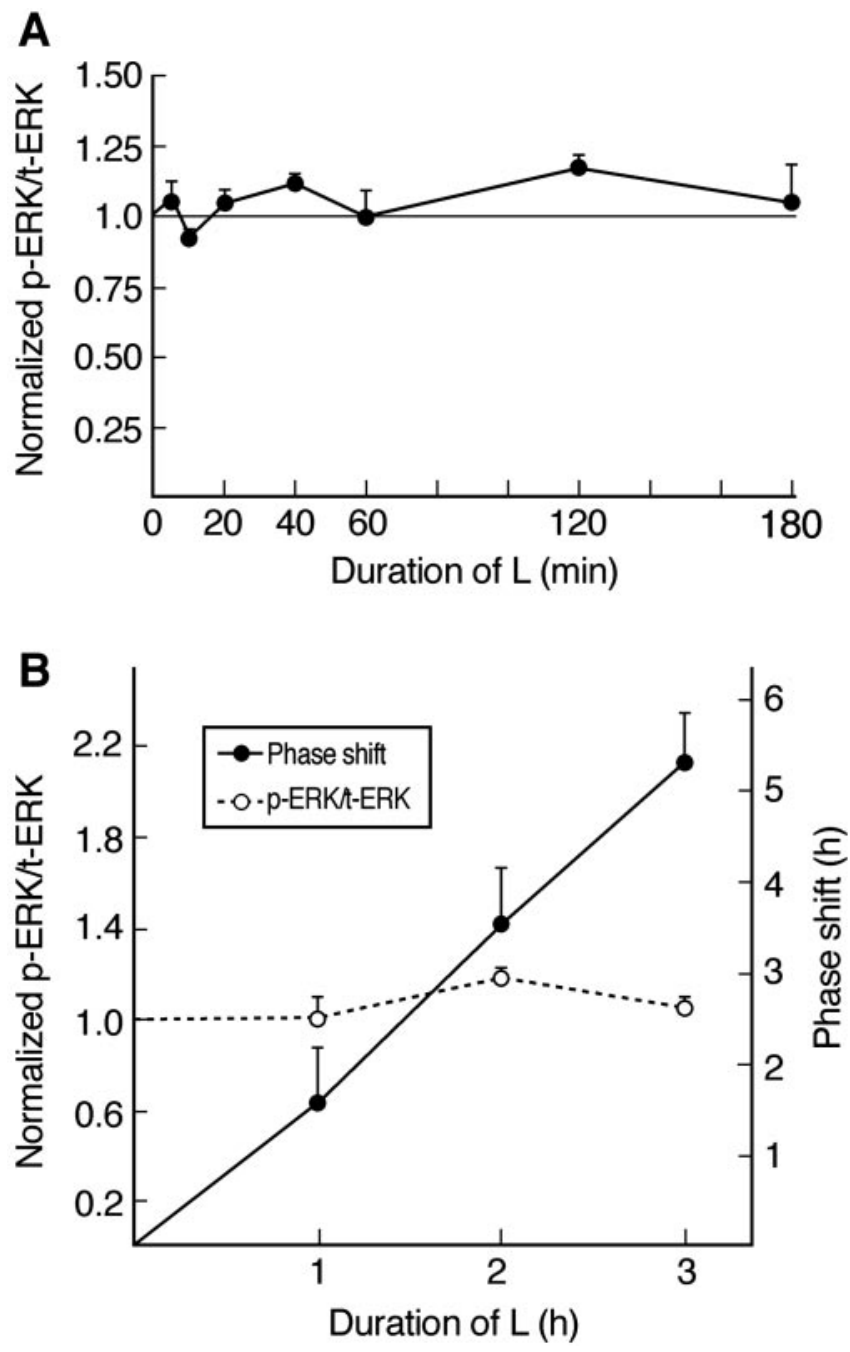

Figure 6. Effects of light pulse duration on ERK and on the melatonin rhythm. A, Exposure to light did not change levels of $p$-ERK. Cells were maintained under LR 12:12 for $5 \mathrm{~d}$ and switched to RR on day 6. Experimental groups were exposed to 5, 10,20,40,60, 120, and 180 min of light pulses, all ending near ZT 16 ( $n=4$ for each time point). Medium was changed immediately preceding the light pulse for all of the groups. Matched control groups ( $n=4$ for each time point) had only their medium changed and were also harvested near ZT 16. Membranes were probed for $p$-ERK and then stripped and reprobed for t-ERK as described in Materials and Methods. $B$, Magnitudes of phase shifts increase with duration of light exposure with no change in p-ERK levels. Cells were maintained under LR 12:12 for $5 d$ and switched to RR on day 6 . Experimental groups were exposed to 1,2 , or $3 \mathrm{hr}$ of $L$ ending at $Z T$ T 16 . Normalized $p$-ERK levels (taken from the experiment shown in $A$ ) and phase shifts of the melatonin rhythm were determined in separate experiments. $p$-ERK (left ordinate) values are shown as open circles and dotted line. Phase shifts (right ordinate) are shown as closed circles and solid line.

examine the effects of both short $(5,10,20$, and $40 \mathrm{~min})$ and long exposures $(1,2$, and $3 \mathrm{hr})$ to light. Separate control groups were used for each duration; their medium was changed and they were harvested at the same times as the experimental groups exposed to light. Medium changes were used to compare the effects of

was an $8 \mathrm{hr}$ interval between medium changes in this group; melatonin output from ZT 8 to 12 was collected together with the melatonin output from ZT 12 to 16 . Half the value obtained is shown at ZT 12, and the other half is shown at ZT 16. Solid lines and closed circles represent results from controls, and dotted lines and open circles represent results from cells with no medium change (No MC) at ZT 12 . Medium contained $0.49 \mu \mathrm{Ci} / \mathrm{ml}^{14} \mathrm{C}$-tryptophan, and blanks (not subtracted) were $103 \pm 5 \mathrm{dpm}$ per well $(n=12)$. 


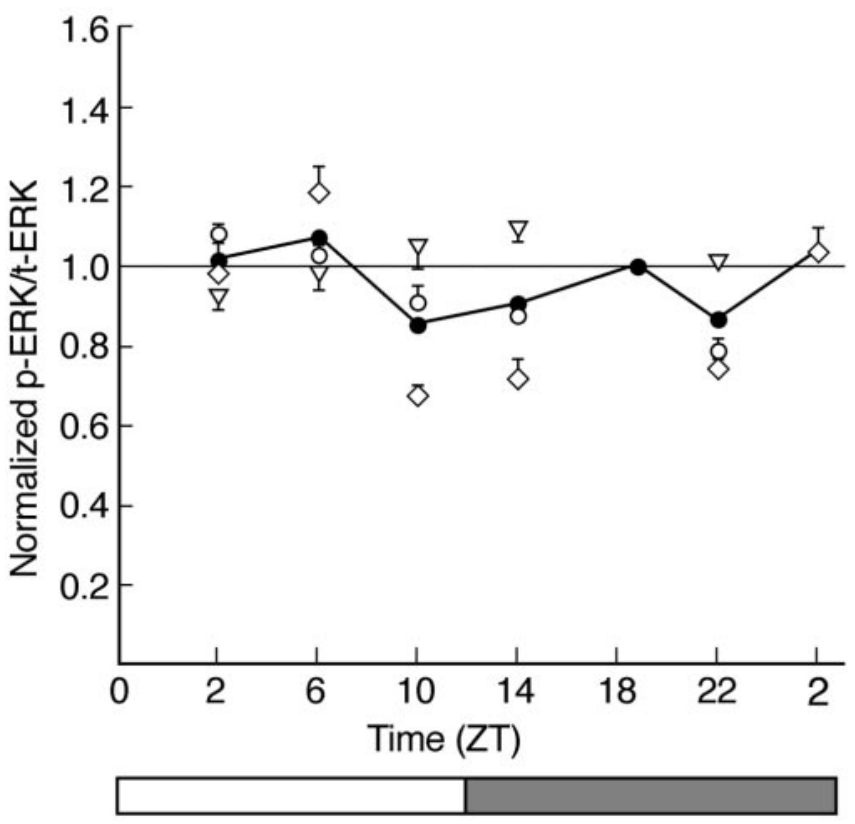

Figure 7. Is there a diurnal rhythm in levels of $p$-ERK? Cells were maintained as usual in LR 12:12 in two different incubators with opposite phases. On day 6, groups were harvested at ZT 2 and 14 (morning and evening) from the two incubators, in $L$ and $R$, respectively, without a medium change. Other groups underwent a last medium change at these times. They were then harvested at $Z T 6$ and 10 in $\mathrm{L}$ from one incubator and at ZT 18 and 22 in R from the other. Thus, all points were harvested at least $4 \mathrm{hr}$ from the last medium change. In one experiment, an additional point was collected at the second ZT 2 to control for possible differences between incubators. Membranes were probed for $\mathrm{p}$-ERK and then stripped and reprobed for t-ERK as described in Materials and Methods. Aliquots of samples from ZT 18 (midnight) were included in every gel, and all other values were normalized to them. Open triangles, open diamonds, and open circles represent values from three separate experiments, each using four wells, for each point. Closed circles and solid line represent the means of the three experiments.

drugs with those of light in the same or similar experiments. Neither short exposure to light nor long exposure to light consistently altered normalized levels of p-ERK relative to controls (Fig. $6 \mathrm{~A})$. Additional experiments with short $(20 \mathrm{~min})$ and long $(4 \mathrm{hr})$ light pulses (starting at ZT 17) confirmed the lack of effect (five experiments with short pulses gave $1.01 \pm 0.01$, and two experiments with long pulses gave $1.03 \pm 0.14$ ).

We also examined the relationship between duration of light exposure and the magnitude of the phase shifts induced. The magnitude of phase shifts increased linearly with increasing duration of light exposure (Fig. $6 \mathrm{~B}$ ). Light pulses of 1,2, and $3 \mathrm{hr}$, all ending at ZT 16, induced phase advances of $+1.6 \pm 0.7,+3.52 \pm$ 0.71 , and $+5.34 \pm 0.7 \mathrm{hr}$, respectively. In contrast, light pulses of the same durations, at the same phase, did not have an increasing effect on levels of p-ERK. Indeed, they had little effect at all (Fig. $6 A, B)$. After light pulses of 1,2 , and $3 \mathrm{hr}$, normalized p-ERK/tERK ratios were $1.0 \pm 0.09,1.18 \pm 0.05$, and $1.04 \pm 0.12$, respectively.

We then examined the relative levels of p-ERK at different times of day, in an LR cycle, in three separate experiments (Fig. 7). There was no apparent diurnal cycle in the normalized p-ERK/t-ERK ratios, nor was there a difference between p-ERK levels in cells exposed to 6 or $10 \mathrm{hr}$ L or to 6 or $10 \mathrm{hr}$ R. Absent a rhythm in LR, we did not test for rhythmicity in RR.

\section{Distribution of ERK and HIOMT in cultured pineal cells}

One possible explanation for the absence of any meaningful response of p-ERK to light is that the fibroblasts or other nonpine-

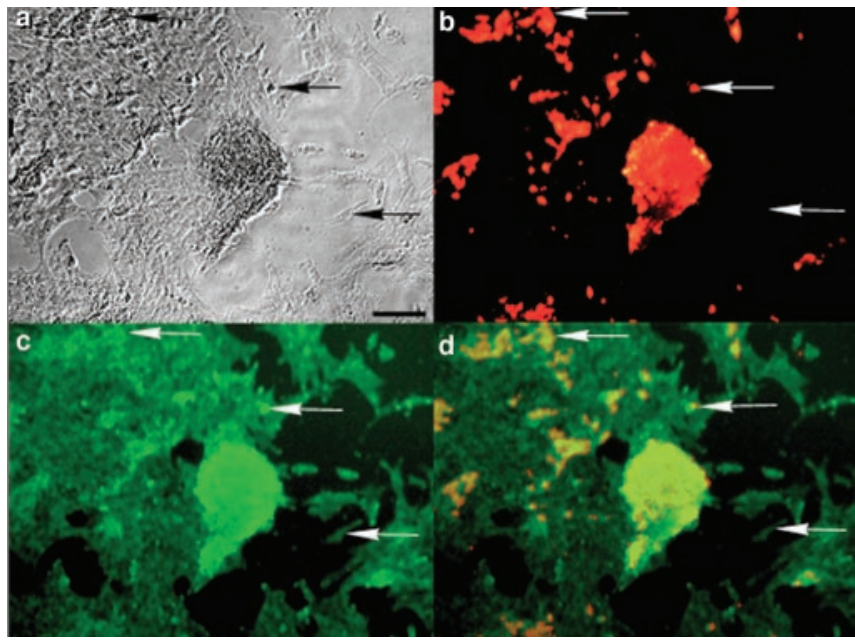

Figure 8. Distribution of HIOMT and ERK among cultured pineal cells. Cells were plated and initially maintained as usual. After $\sim 45 \mathrm{hr}$ in culture, however, they were fixed at ZT 12 and analyzed by immunocytochemistry as described in Materials and Methods. A, Phase-contrast image of a field of pineal cells at $20 \times$ magnification. Pinealocytes aggregate and form clumps (as seen in the middle of the field) on top of a feeder layer of fibroblasts. At this time after plating, individual pinealocytes and fibroblasts can still be seen, as can relatively small clumps (compared with those in mature cultures) of putative pinealocytes and incipient clumps on fibroblasts. The arrows are the same in each panel. Topmost arrow points toward an incipient pinealocyte clump. Middle arrow points to a few individual pinealocytes clumped together. Lowest arrow points to an individual fibroblast. Scale bar, $50 \mu \mathrm{m}$. B, Same field showing cells immunolabeled for the pinealocyte marker HIOMT. Melatonin-producing cells are red; fibroblasts are not visible. C, Same field showing cells immunolabeled for ERK. All cells in the field are green and contain ERK. D, Overlay showing double-labeled cells (seen in yellow).

alocytes in the cultures (there are a few erythrocytes and glial cells also present) are so predominant as to obscure any response from the pinealocytes. If $90 \%$ of the cells were nonpinealocytes, for example, then a photoinduced $50 \%$ reduction of p-ERK levels restricted to the pinealocytes would not have been visible. Alternatively, if $75 \%$ of the cells were pinealocytes, then a rhythm with an amplitude of $20 \%$ would not have been visible. We could not determine the precise ratio of pinealocytes to nonpinealocytes in mature cultures (day 6). There, the putative pinealocytes sit on confluent fibroblasts in large clumps containing thousands of cells that are macroscopically visible, often $1 \mathrm{~mm}$ in diameter (Zatz et al., 1988). Such clumps interfere with antibody access and immunocytochemical procedures, and make it difficult to disperse all the cells in a well and recover them intact, undisturbed, and in the original proportions. Consequently, we examined younger cultures, $\sim 45 \mathrm{hr}$ after plating (day 3 ), when the fibroblasts had not yet achieved full confluence and clumping of pinealocytes had only begun. There were still many single pinealocytes, or clumps with small numbers of pinealocytes, visible, often sitting on fibroblasts (Fig. 8a). The proportion of pinealocytes to nonpinealocytes in these cultures is much smaller than in mature cultures, because the cells are still multiplying, and the number of pinealocytes in the small clumps is only a small fraction of the number that are present later in the very large clumps.

Pinealocytes, by definition, make melatonin and contain the organotypic enzyme HIOMT. They can be identified (Voisin et al., 1988) by their specific immunoreactivity to anti-HIOMT antibody (Fig. 8b). By visual estimates, more than half the cells were positive for HIOMT on day 3. All of the cells, however, were positive for ERK (Fig. 8c). All of HIOMT-containing cells also contained ERK (Fig. $8 d$ ). Control experiments omitting either of the primary or secondary antibodies showed no significant back- 
ground staining. The thousands of cells per clump, and the many clumps, in the mature cultures (day 6), however, are likely to result in the pinealocytes being the predominant cell type there.

\section{Discussion}

We first sought a correlation between the ability of PD 98059 and U0126, two well known inhibitors of MAPK/ERK activation (Alessi et al., 1995; Favata et al., 1998; Davies et al., 2000), to reduce p-ERK levels and induce phase shifts in the melatonin rhythm. Both drugs markedly reduced levels of p-ERK (Figs. $1 \mathrm{~A}$, 2A). The less potent drug, PD 98059 (Alessi et al., 1995; Dudley et al., 1995; Favata et al., 1998), did induce light-like phase shifts at reasonable concentrations $\left(\sim 10 \times\right.$ reported $\left.\mathrm{IC}_{50}\right)$ (Figs. $\left.1 C, D\right)$, supporting (indeed, generating) the hypothesis that a reduction in ERK activity is sufficient for photoentrainment. The more potent drug, U0126 (Favata et al., 1998), was expected to confirm the result and strengthen the hypothesis. Instead, U0126 markedly reduced p-ERK levels with no induction of phase shifts (Fig. $2 B$ ) even at $\sim 100 \times$ reported $\mathrm{IC}_{50}$, thereby putting the hypothesis into question.

The p-p38 inhibitor, SB203580 (Cuenda et al., 1995), should have had no effect on the phase of the melatonin rhythm or on p-ERK levels. Instead, it induced dark-like phase shifts at concentrations $\left(\sim 20 \times\right.$ reported $\left.\mathrm{IC}_{50}\right)$ that would be expected to markedly inhibit p-p38 activity without much effect on p-ERK levels (Fig. 3A). Xenopus retina apparently shows similar effects (Hasegawa et al., 1999). At still higher concentrations of SB203580 $\left(\sim 200 \times\right.$ reported $\left.\mathrm{IC}_{50}\right)$, where specificity is likely to be lost, the drug induced very strong dark-like phase shifts and markedly reduced p-ERK levels (Figs. $3 \mathrm{~B}, \mathrm{C}$ ). This reduction in p-ERK levels, comparable to those seen with PD 98059 and U0126, was not sufficient, however, to induce light-like phase shifts or reduce the magnitude of the dark-like phase shifts induced by the drug. Thus, results with the p-p38 inhibitor, SB203580, further undermine the hypothesis that reductions in ERK activation induce light-like phase shifts. The ability of SB203580 to induce dark-like phase shifts might encourage the alternate hypothesis that changes in p-p38 activity are involved in photoentrainment, as has been suggested (Hayashi et al., 2003). However, the likely loss of specificity (Hasegawa and Cahill, 2000) at the high concentrations that gave the strongest effects, and our experiences here with agents that change p-ERK levels, indicate that this hypothesis too should be approached with caution.

The hypothesis tested requires that perturbations that markedly reduce p-ERK levels induce light-like phase shifts. We found five perturbations that reduced p-ERK levels. Of these, two induced light-like phase shifts (PD 98059 and caffeine), one induced dark-like phase shifts (SB203580), and two did not induce phase shifts at all (U0126 and omitting the medium exchange). In the other direction, perturbations that increase p-ERK levels should induce dark-like phase shifts. Despite the clear increases in p-ERK levels after addition of high concentrations of serum, dark-like phase shifts, indeed, phase shifts of any kind, did not occur (Fig. 4). Taken together, these results argue strongly against the hypothesis that reduction (or increases) in p-ERK levels are necessary or sufficient for phase shifts. It should be noted that these are not negative results or "failures to find." Instead, combinations of positive results were found that, taken together, belie the requirements and predictions of the hypothesis tested (Zatz, 2002).

We found no significant changes in p-ERK levels in response to light pulses that are effective in inducing phase shifts (Fig. 6). Neither did we find a diurnal rhythm in p-ERK levels (Fig. 7).
Our results and interpretations differ from those of two other groups. In mice, light pulses in vivo were reported to increase levels of p-ERK in the suprachiasmatic nucleus (Obrietan et al., 1998), and pretreatment with U0126 reduced the magnitude of light-induced phase shifts of the rhythm in locomotor activity (Butcher et al., 2002). It is difficult to assess the relevance of these results, however, because the complexity of the circuitry and interactions of the neurons in the suprachiasmatic nucleus make its clock far more promiscuously responsive to stimulation by various neurotransmitters, peptides, and transduction pathways (Gillette and Mitchell, 2002) than is the chick pineal. Of greater relevance are the results and interpretations from another group (Sanada et al., 2000; Hayashi et al., 2001) who reported a rapid and transient decrease in activated MAPK/ERK in chick pineal glands in response to light. These changes are in the opposite direction from those reported in mouse brain. The authors suggested that such decreases mediate photoentrainment, essentially the hypothesis tested here.

We do not know the basis for the differences in our results and those of Sanada et al. (2000) and Hayashi et al. (2001). There were, however, a number of differences in experimental design and methodology. We used dispersed cultured cells exclusively. They used whole glands, either ex vivo or in culture. Consequently, harvest procedures differed. We stopped ongoing processes almost instantaneously by adding Laemmli buffer, whereas they had first to dissect out glands in dim red light or in white light before pooling and homogenizing them. We do not know whether the rapid changes in p-ERK levels that we observed in response to mechanical agitation (Fig. 5A) are relevant to the differences in results. Sanada et al. (2000) immunoprecipitated pineal homogenates with an antibody that recognizes t-ERK and then ran the samples on gels and probed using an antiphosphotyrosine antibody, whereas Hayashi et al. (2001) immunoblotted pineal homogenates directly and used a primary antibody against p-ERK. We always treated cells directly with Laemmli buffer, ran the samples, and detected p-ERK by immunoblot using primary antibody against p-ERK.

It is notable that the reductions in activated MAPK/ERK reported by Sanada et al. (2000) and Hayashi et al. (2001) were transient: maximal in $\sim 0.5 \mathrm{hr}$ and essentially gone in $1 \mathrm{hr}$. Our experiments demonstrated that the size of the phase shift induced by light increases linearly as the duration of the light pulse increases from 1 to 2 to $3 \mathrm{hr}$ (Fig. $6 \mathrm{~B}$ ). Consequently, the level of an activated transducer would not be expected to return to baseline in $1 \mathrm{hr}$, yet somehow mediate a continuing, linear increase in the magnitude of the downstream effect for an additional $2 \mathrm{hr}$. A time course more like that for the response of p-ERK levels to PD 98059 would be expected. At concentrations that induced phase shifts smaller than, or comparable with, those induced by light pulses, PD 98059 caused a marked, rapid, and sustained reduction in p-ERK levels. Only one of our perturbations, in effect, reduced p-ERK levels transiently: omitting the usual replacement of spent with fresh medium. This perturbation did not induce phase shifts in the melatonin rhythm (Fig. 5B). Thus, a transient reduction in levels of activated MAPK/ERK similar to that reported, but achieved in a different way, was not able to phase shift the circadian clock. Moreover, effects of mere medium change emphasize the importance of comprehensive controls for, and cautious interpretation of, changes in MAPK/ERK.

Our exclusive use of dispersed cell cultures might provide an explanation for the absence of a light effect on levels of activated MAPK/ERK in our experiments, as compared with those of Sanada et al. (2000) and Hayashi et al. (2001). It could be postu- 
lated that, in contrast to whole glands, the population of cells in our wells consists predominantly of fibroblasts or other nonpinealocytes or nonphotosensitive cells. Because these cells would then provide the bulk of the p-ERK in the wells and would not be expected to respond to light, p-ERK levels in the well as a whole might not change much despite their significantly reduced levels in the photosensitive pinealocytes. This explanation is belied, however, by our results from immunocytochemistry (Fig. 8), taken together with the known changes in organization and cell ratios with time in culture (Zatz et al., 1988). These results indicate that the proportion of pinealocytes was sufficiently high to provide a reliably measurable effect on the p-ERK levels of the wells if their p-ERK levels had changed significantly. The same is true for detection of a sizable diurnal rhythm. That a lightinduced change in p-ERK levels was present in pinealocytes but obscured by the high levels of p-ERK in nonpinealocytes seems unlikely.

Furthermore, in addition to the postulate of obscured signal in response to light, it would be necessary, to maintain the hypothesis that changes in p-ERK levels are necessary and sufficient for photoentrainment, to postulate additional properties of the system: that both nonpinealocytes and pinealocytes are sensitive to PD 98059, but that pinealocytes are selectively refractory to the effects of U0126 on p-ERK and the melatonin rhythm; that, despite this refractoriness, there is still no detectable p-ERK signal from pinealocytes when U0126 reduces p-ERK levels from the other cell types in the well to undetectable levels; that the pinealocytes are sensitive to the effects of SB203580 on p-p38, but that only the nonpinealocytes carry the effect on p-ERK; that the transient effects of medium change are selectively expressed by nonpinealocytes but would induce phase shifts if they were expressed by pinealocytes; and that the increases in p-ERK levels induced by serum are selectively expressed by nonpinealocytes but would induce phase shifts if they were expressed by pinealocytes. That all these post hoc properties of the system would hold seems far fetched.

In conclusion, we have tested the requirements and predictions of the hypothesis that decreases (or increases) in levels of activated MAPK/ERK are necessary and/or sufficient for entrainment of chick pineal cells in culture. The weight of the evidence strongly favors the conclusion that they are not. However, we have not addressed or ruled out other hypotheses, even related hypotheses, especially in other systems. In addition to the possibility of a role for MAPK/ERK or related pathways in entrainment of the circadian clocks in the suprachiasmatic nucleus, there is evidence suggesting roles for MAPK/ERK in clock-controlled output pathways in chick retinal cones (Ko et al., 2001) and fly brains (Williams et al., 2001). The ubiquity, the many variants, the complexity, and the ready responsiveness of MAPK/ERK and related systems, however, make it advisable to use caution in the attribution of specific physiologic roles to one of these pathways.

\section{References}

Alessi DR, Cuenda A, Cohen P, Dudley DT, Saltiel AR (1995) PD 0980509 is a specific inhibitor of the activation of mitogen-activated protein kinase kinase in vitro and in vivo. J Biol Chem 270:27489-27494.

Arbabi S, Maier RV (2002) Mitogen-activated protein kinases. Crit Care Med 30:S74-S79.

Butcher GQ, Dziema H, Collamore M, Burgoon PW, Obrietan K (2002) The p42/44 mitogen-activated protein kinase pathway couples photic input to circadian clock entrainment. J Biol Chem 277:29519-29525.

Cermakian N, Pando MP, Thompson CL, Pinchak AB, Selby CP, Gutierrez L, Wells DE, Cahill GM, Sancar A, Sassone-Corsi P (2002) Light induction of a vertebrate clock gene involves signaling through blue-light receptors and MAP kinases. Curr Biol 12:844-848.
Cobb MH (1999) MAP kinase pathways. Prog Biophys Mol Biol 77:479-500.

Cuenda A, Rouse J, Doza YN, Meier R, Cohen P, Gallagher TF, Young PR, Lee JC (1995) SB 203580 is a specific inhibitor of a MAP kinase homologue which is stimulated by cellular stresses and interleukin-1. FEBS Lett 364:229-233.

Davies SP, Reddy H, Caivano M, Cohen P (2000) Specificity and mechanism of action of some commonly used protein kinase inhibitors. Biochem J 351:95-105.

Deguchi T (1979) A circadian oscillator in cultured cells of chicken pineal gland. Nature 282:94-97.

Dudley DT, Pang L, Decker SJ, Bridges AJ, Saltiel AR (1995) A synthetic inhibitor of the mitogen-activated protein kinase cascade. Proc Natl Acad Sci USA 92:7686-7689.

Favata MF, Horiuchi KY, Manos EJ, Daulerio AJ, Stradley DA, Feeser WS, Van Dyk DE, Pitts WJ, Earl RA, Hobbs F, Copeland RA, Magolda RL, Scherle PA, Trzaskos JM (1998) Identification of a novel inhibitor of mitogen-activated protein kinase kinase. J Biol Chem 273:18623-18632.

Gillette MU, Mitchell JW (2002) Signaling in the suprachiasmatic nucleus: selectively responsive and integrative. Cell Tissue Res 309:99-107.

Hasegawa M, Cahill GM (2000) A role for stress-activated protein kinases (SAPKs) in the photoreceptor circadian clock. Invest Ophthalmol Vis Sci Abstr 41:S26.

Hasegawa M, Hattar S, Eskin A, Cahill GM (1999) An inhibitor of p38 MAP kinase resets the circadian oscillator in cultured Xenopus retinal photoreceptors. Int Cong Chronobiol Abstr 70.

Hayashi Y, Sanada K, Fukada Y (2001) Circadian and photic regulation of MAP kinase by Ras- and protein phosphatase-dependent pathways in the chick pineal gland. FEBS Lett 491:71-75.

Hayashi Y, Sanada K, Hirota T, Shimizu F, Fukada Y (2003) p38 Mitogenactivated protein kinase regulates oscillation of chick pineal circadian clock. J Biol Chem 278:25166-25171.

Johnson GL, Lapadat R (2002) Mitogen-activated protein kinase pathways mediated by ERK, JNK, and p38 protein kinases. Science 298:1911-1912.

Johnson ML, Frasier SG (1985) Nonlinear least squares analysis. Methods Enzymol 117:301-342.

Ko GY, Ko ML, Dryer SE (2001) Circadian regulation of cGMP-gated cationic channels of chick retinal cones: Erk MAP kinase and $\mathrm{Ca}^{2+} /$ calmodulin-dependent protein kinase II. Neuron 29:255-266.

Mackova M, Man JR, Chik CL, Ho AK (2000) p38 ${ }^{\text {MAPK }}$ inhibition enhances basal and norepinephrine stimulated $\mathrm{p} 42 / 44^{\mathrm{MAPK}}$ phosphorylation in rat pinealocytes. Endocrinology 141:4202-4208.

Mitra G, Weber M, Stacey D (1993) Multiple pathways for activation of MAP kinases. Cell Mol Biol Res 39:517-523.

Natesan A, Geetha L, Zatz M (2002) Rhythm and soul in the avian pineal. Cell Tissue Res 309:35-45.

Obrietan K, Impey S, Storm DR (1998) Light and circadian rhythmicity regulate MAP kinase activation in the suprachiasmatic nuclei. Nat Neurosci 1:693-700.

Pearson G, Robinson F, Gibson TB, Xu B, Karandikar M, Berman K, Cobb MH (2001) Mitogen-activated protein (MAP) kinase pathways: regulation and physiological functions. Endocr Rev 22:153-183.

Sanada K, Hayashi Y, Harada Y, Okano T, Fukada Y (2000) Role of circadian activation of mitogen-activated protein kinase in chick pineal clock oscillation. J Neurosci 20:986-991.

Straume M, Frasier-Cadoret SG, Johnson ML (1991) Least squares analysis of fluorescence data. In: Topics in fluorescence spectroscopy (Lakowicz JR, ed), pp 177-240. New York: Plenum.

Su B, Karin M (1996) Mitogen-activated protein kinase cascades and regulation of gene expression. Curr Opin Immunol 8:402-411.

Takahashi JS, Murakami N, Nikaido SS, Pratt BL, Robertson LM (1989) The avian pineal, a vertebrate model system of the circadian oscillator: cellular regulation of circadian rhythms by light, second messengers, and macromolecular synthesis. Recent Prog Horm Res 45:279-348.

Troppmair J, Bruder JT, Munoz H, Lloyd PA, Kyriakis J, Banerjee P, Avruch J, Rapp UR (1994) Mitogen-activated protein kinase/extracellular signal-regulated protein kinase activation by oncogenes, serum, and 12$O$-tetradecanoylphorbol-13-acetate requires raf and is necessary for transformation. J Biol Chem 269:7030-7035.

Valjent E, Caboche J, Vanhoutte P (2001) Mitogen-activated protein kinase/ extracellular signal-regulated kinase induced gene regulation in brain. A molecular substrate for learning and memory? Mol Neurobiol 23:85-101. 
Voisin P, Guerlotte J, Collin JP (1988) An antiserum against chicken hydroxyindole-O-methyltransferase reacts with the enzyme from pineal gland and retina and labels pineal modified photoreceptors. Mol Brain Res 4:53-61.

Williams JA, Su HS, Bernards A, Field J, Sehgal A (2001) A circadian output in Drosophila mediated by neurofibromatosis-1 and Ras/MAPK. Science 293:2251-2256.

Yokota S, Yamamoto M, Moriya T, Akiyama M, Fukunaga K, Miyamoto E, Shibata S (2001) Involvement of calcium-calmodulin protein kinase but not mitogen-activated protein kinase in light-induced phase delays and Per gene expression in the suprachiasmatic nucleus of the hamster. J Neurochem 77:618-627.

Zatz M (1996) Melatonin rhythms: trekking toward the heart of darkness in the chick pineal. Cell Dev Biol 7:811-820.
Zatz M (2002) Editorial: Ya gotta believe! J Biol Rhythms 17:3.

Zatz M, Heath III JR (1995) Calcium and photoentrainment in chick pineal cells revisited: effects of caffeine, thapsigargin, EGTA, and light on the melatonin rhythm. J Neurochem 65:1332-1341.

Zatz M, Wang HM (1991) High salt mimics the effects of light pulses on the circadian pacemaker in cultured chick pineal cells. Am J Physiol 260:R769-776.

Zatz M, Mullen DA, Moskal JR (1988) Photoendocrine transduction in cultured chick pineal cells: effects of light, dark, and potassium on the melatonin rhythm. Brain Res 438:199-215.

Zatz M, Gastel JA, Heath JR III, Klein DC (2000) Chick pineal melatonin synthesis: light and cyclic AMP control abundance of serotonin $N$-acetyltransferase protein. J Neurochem 74:2315-2321. 